\title{
Dinoflagellates and ciliates at Helgoland Roads, North Sea
}

\author{
Martin Günther Joachim Löder • Alexandra Claudia Kraberg • \\ Nicole Aberle $\cdot$ Silvia Peters $\cdot$ Karen Helen Wiltshire
}

Received: 19 July 2010/Revised: 6 December 2010/ Accepted: 27 December 2010/Published online: 19 January 2011

(C) Springer-Verlag and AWI 2011

\begin{abstract}
A monitoring programme for microzooplankton was started at the long-term sampling station "Kabeltonne" at Helgoland Roads $\left(54^{\circ} 11.3^{\prime} \mathrm{N} ; 7^{\circ} 54.0^{\prime} \mathrm{E}\right)$ in January 2007 in order to provide more detailed knowledge on microzooplankton occurrence, composition and seasonality patterns at this site and to complement the existing plankton data series. Ciliate and dinoflagellate cell concentration and carbon biomass were recorded on a weekly basis. Heterotrophic dinoflagellates were considerably more important in terms of biomass than ciliates, especially during the summer months. However, in early spring, ciliates were the major group of microzooplankton grazers as they responded more quickly to phytoplankton food availability. Mixotrophic dinoflagellates played a secondary role in terms of biomass when compared to heterotrophic species; nevertheless, they made up an intense late summer bloom in 2007. The photosynthetic ciliate Myrionecta rubra bloomed at the end of the sampling period. Due to its high biomass when compared to crustacean plankton especially during the spring bloom, microzooplankton should be regarded as the more important phytoplankton grazer group at Helgoland Roads. Based on these results, analyses of biotic and abiotic factors driving microzooplankton composition and abundance are necessary for a full understanding of this important component of the plankton.
\end{abstract}

Communicated by H.-D. Franke.

M. G. J. Löder $(\bowtie) \cdot$ A. C. Kraberg · N. Aberle · S. Peters ·

K. H. Wiltshire

Biologische Anstalt Helgoland, Alfred Wegener Institute for Polar and Marine Research, Marine Station, POB 180, 27483 Helgoland, Germany

e-mail: Martin.Loeder@awi.de
Keywords Dinoflagellates - Ciliates · North Sea · Monitoring · Helgoland Roads · Seasonality

\section{Introduction}

Marine research has a long tradition on Helgoland. Water temperature has been measured at the Helgoland Roads long-term station "Kabeltonne" $\left(54^{\circ} 11.3^{\prime} \mathrm{N} ; 7^{\circ} 54.0^{\prime} \mathrm{E}\right)$ since 1873 (Wiltshire and Manly 2004), and biological, chemical and physical parameters have been recorded continuously on a work-daily basis since 1962 (Franke et al. 2004). This makes the Helgoland long-term data series one of the longest and most detailed aquatic data sets. Unique to this data set are the phytoplankton species numbers counted work-daily to species level wherever possible (Wiltshire and Dürselen 2004). Since 1975, the time series also includes meso- and macrozooplankton determined to species level three times per week (Greve et al. 2004). Thus, the time series provides an excellent basis for analyses of long-term trends including changes evinced in the North Sea pelagic system over the recent decades (Schlüter et al. 2008; Wiltshire et al. 2008). Furthermore, it is a very important basis for the parameterisation and validation of mathematical ecosystem models and is invaluable in biodiversity and global change considerations (Wirtz and Wiltshire 2005). However, one important group of planktonic organisms is under-represented in the long-term series so far-the microzooplankton. Although data on heterotrophic dinoflagellates exist in the data set, they were recorded with varying degrees of accuracy (Wiltshire and Dürselen 2004) and did not always mirror the diversity in species composition (Hoppenrath 2004). Long-term data on ciliates, another crucial microzooplankton group, are totally lacking at Helgoland Roads. 
The term microzooplankton refers to the size fraction of heterotrophic planktonic organisms between 20 and $200 \mu \mathrm{m}$. Consisting of a diverse array of protozoa and metazoa, its numerically most important components are heterotrophic dinoflagellates and ciliates (Capriulo et al. 1991). Recent research, for example, by Landry and Calbet (2004), demonstrated the fundamental importance of microzooplankton as phytoplankton grazers. They showed that grazing by microzooplankton can be as high as $60-75 \%$ of the daily phytoplankton production. Furthermore, results indicate that microzooplankton tends to surpass mesozooplankton as primary consumers (Sherr and Sherr 2007). A meta-analysis of Calbet and Landry (2004) revealed that microzooplankton grazing can be responsible for $60 \%$ of phytoplankton mortality in coastal and estuarine environments (comparable to Helgoland Roads in terms of chlorophyll $a$ concentration). We realised from observations that microzooplankton could also potentially be the most important grazer group in waters at Helgoland. Therefore, the aim of this study was to supplement the regular plankton monitoring series at Helgoland with an intensive monitoring of the microzooplankton. Investigations into species composition and seasonality of this important functional grazer group (dinoflagellates and ciliates) on a more regular basis will provide vital baseline data for studies of long-term changes in the microzooplankton community and the pelagic system at Helgoland.

\section{Materials and methods}

A 2.5-year microzooplankton monitoring programme has been carried out at Helgoland Roads to investigate the abundance of dinoflagellates and ciliates in the Southern North Sea. This monitoring hoped to establish a higher taxonomic resolution and to improve the evaluation of biomass for single taxa of microzooplankton.

From January 2007 until June 2009, samples were taken once a week at the "Kabeltonne" site $\left(54^{\circ} 11.3^{\prime} \mathrm{N} ; 7^{\circ} 54.0^{\prime} \mathrm{E}\right)$ at Helgoland. These data supplemented the routine sampling programme, which is carried out week-daily and for which plankton samples are fixed with a weak neutral Lugol's solution (final concentration 0.5\%) (Wiltshire et al. 2008). Although dinoflagellates are counted within the long-term programme, the taxonomic focus lies on phytoplankton groups such as diatoms. Due to the time-consuming counting procedure for phytoplankton and the high frequency of samples (work-daily), rare, small or uncommon dinoflagellate taxa are inevitably neglected or categorised into size classes. The present study investigated such under-represented species more intensely during the 2.5-year microzooplankton monitoring. Apart from three ciliates that have recently been included in the counting programme (Myrinecta rubra 1999, Laboea strobila 2007, Mesodinium pulex 2008), no ciliate species had continuously been recorded previously at Helgoland. Therefore, the new microzooplankton monitoring presented here was to provide completely new information on ciliate biomass and seasonality patterns at a hitherto unavailable taxonomic resolution.

The loss of microzooplankton species due to fixative problems has often been discussed in the literature (Stoecker et al. 1994). Thus, we diverged from the neutral fixative used for the long-term monitoring and used acidic Lugol's solution (final concentration 2\%, Throndsen 1978), as this is the standard fixative used in most studies on microzooplankton composition. The concentration we used has been proven to be the best compromise for both conserving higher concentrations of ciliates and preventing shrinkage of cells (Stoecker et al. 1994). A subsample of $250 \mathrm{~mL}$ was fixed immediately. Samples were stored in the cold and dark, and then $50 \mathrm{~mL}$ of the sample were settled for $24 \mathrm{~h}$ and counted under an inverted microscope (Zeiss Axiovert 135) using the Utermöhl method (Utermöhl 1958, Lund et al. 1958). At least half of the surface of the sedimentation chamber or the whole chamber was counted out at 200-fold magnification, thus reducing counting biases against rare species. Identification of naked dinoflagellates and especially of ciliates in Lugol's solution-preserved samples is often difficult below genus level (Johansson et al. 2004), even with the modified fixation method applied here. Therefore, problematic ciliates and dinoflagellates were identified to genus level or, otherwise, pooled into size-dependent groups and "morphotypes", based on their similar shape. Mixotrophy of the ciliates was not measured; thus, we have no exact data on the percentage of mixotrophic ciliates in the samples. However, to date, all mixotrophic ciliates have been shown to be phagotrophic (Sherr and Sherr 2002) and consequently all ciliates except Myrionecta rubra could be considered heterotrophic (Johansson et al. 2004). The latter species acts essentially as a phototroph (Montagnes et al. 2008), but as recent studies have shown that it also has phagotrophic capabilities (Park et al. 2007), we thus recorded it as mixotrophic.

As most chloroplast-bearing dinoflagellates are also capable of mixotrophic nutrition via phagotrophy (Du Yoo et al. 2009), these were considered to be potential grazers with more or less marked phagotrophic capabilities. The identification of dinoflagellates was primarily based on Dodge (1982), Tomas (1996) and Hoppenrath et al. (2009). Ciliates were determined based on Kahl (1932), Carey (1992) and Montagnes (2003).

As an additional new feature compared to the regular longterm series, each taxon recorded during counting was documented by an image (archived online at http://planktonnet. 
awi.de). These images, used for subsequent biovolume estimations, were also a useful tool for the documentation of rare and prior unrecorded species and for subsequent taxon assignments.

After measurement of the linear dimensions of the cells in the images, the biovolume of each taxon was calculated using the geometric models described by Hillebrand et al. (1999). The biovolume was converted into carbon using the conversion factor given by Putt and Stoecker (1989) for ciliates and Menden-Deuer and Lessard (2000) for dinoflagellates. The carbon content of the large "gelatinous" dinoflagellate Noctiluca scintillans was estimated according to the biovolume-carbon relationship given by Tada et al. (2000) after correction for shrinkage due to fixation (Beran et al. 2003). Carbon concentrations $\left[\mu \mathrm{gC} \mathrm{L}^{-1}\right]$ of dinoflagellates and ciliates are hereinafter also referred to as biomass or carbon biomass.

In vivo fluorescence as a proxy for phytoplankton biomass is measured on a work-daily basis (Algae Analyser, BBE Moldaenke, Kiel, Germany) as part of the routine monitoring at Helgoland Roads. These data were used as a rough indicator for autotrophic biomass and for the purpose of illustration of phytoplankton food availability and are presented in the results.

For the evaluation of the microzooplankton monitoring data, we compared them with the available data of the Helgoland Roads long-term data set on plankton. After evaluation of the literature on the quality of this data set (Wiltshire and Dürselen 2004) and the results of an unpublished revision of the data by S. Peters and M. Scharfe, two species that cannot be confused with other taxa were identified for the comparison: the dinoflagellate Noctiluca scintillans and the ciliate Myrionecta rubra.

\section{Results and discussion}

\section{5-year microzooplankton monitoring}

During the 2.5-year monitoring programme, 122 different taxa of dinoflagellates and ciliates were recorded (Tables 1 , 2). Each group of organisms contributed roughly $50 \%$ to the total number of taxa.

Sixty dinoflagellate taxa were recorded. Thirty-nine of them could be regarded as truly heterotrophic because they lacked chloroplasts, and the remainder was considered to be mixotrophic (Table 1). The ciliates found comprised 62 taxa. Due to their phagotrophic feeding capabilities, all ciliates were considered heterotrophic, with the exception of Myrionecta rubra (mixotrophic).

Heterotrophic dinoflagellates were always present in carbon concentrations between 0.5 and $272 \mu \mathrm{gC} \mathrm{L} \mathrm{L}^{-1}$, and mixotrophic dinoflagellates in carbon concentrations between 0.2 and $422 \mu \mathrm{gC} \mathrm{L}{ }^{-1}$. During the 2.5 -year period, the most important orders of dinoflagellates in terms of carbon biomass were mixotrophic Gymnodiniales (31\%), followed by Noctilucales (25\%), heterotrophic Gymnodiniales $(17 \%)$ and mixotrophic (14\%) as well as heterotrophic Peridiniales (7\%) (Fig. 1, left panel). Prorocentrales, Gonyaulacales and Dinophysiales played only a minor role from a carbon biomass perspective (1-3\%).

Dinoflagellates closely followed the chlorophyll $a$ development in spring, and biomass started to increase from March onwards (Fig. 2). Peaks in biomass often coincide with those of chlorophyll $a$ or succeeded them, suggesting close coupling between prey availability and predator biomass.

Maximum values always occurred during the summer months (June-August) when Noctiluca scintillans, Gyrodinium spp. and Protoperidinium spp. occurred together. Especially during this period, we detected high fluctuations in chlorophyll $a$ and dinoflagellate biomass (summer 2007/2008 and spring 2009), suggesting high growth and mortality rates. However, these patterns did not reflect natural growth or mortality and could be traced back to variations in water bodies due to, for example, tidal currents and changing wind directions which were visible in abrupt changes in salinity and nutrients (data not shown). Towards winter and in tandem with decreasing chlorophyll $a$ concentrations, heterotrophic dinoflagellate biomass reached its minimum suggesting close coupling with phytoplankton food availability. Outliers in biomass of heterotrophic dinoflagellates in December 2007 and January 2008 stem from the presence of single cells of $N$. scintillans. During the investigation period, mixotrophic dinoflagellates (Fig. 2) usually played a minor role compared to heterotrophic species $\left(0.2-30 \mu \mathrm{gC} \mathrm{L}^{-1}\right)$. Only in summer $2007 \mathrm{did}$ they form an intense bloom from end of July to mid of October, thereby greatly exceeding the biomass of heterotrophic dinoflagellates (Fig. 2) and reaching values up to $422 \mu \mathrm{gC}$ $\mathrm{L}^{-1}$. The bloom was first composed mainly of Lepidodinium chlorophorum as well as Scrippsiella/Pentapharsodinium sp. and Prorocentrum triestinum in lower densities. From mid September onwards, the bloom consisted mainly of Akashiwo sanguinea. During the rest of the sampling period, mixotrophic dinoflagellates were usually present in much lower concentrations than heterotrophic ones.

Ciliated protozoa were present throughout the time of monitoring with concentrations varying between 0.2 and $106 \mu \mathrm{gC} \mathrm{L}^{-1}$ (Fig. 3). In terms of carbon biomass, the ciliate order Strombidiida played the most important role during the monitoring programme being responsible for more than half of the biomass (51\%), followed by M. rubra (23\%) and then Choreotrichida (7\%) and Haptorida (6\%) (Fig. 1, right panel). Cyclotrichiida, Tintinnida and Prorodontida played a certain role $(2-4 \%)$. The remaining 
Table 1 Dinoflagellate taxa and their seasonality as well as maximum cell concentrations, the mean biovolume and mean carbon content of each taxon as recorded during the 2.5 years of microzooplankton monitoring

\begin{tabular}{|c|c|c|c|c|c|c|}
\hline Dinoflagellates & $\begin{array}{l}\text { Assigned } \\
\text { trophy }\end{array}$ & $\begin{array}{l}\text { Observed } \\
\text { seasonality }\end{array}$ & $\begin{array}{l}\text { Maximum } \\
\left(\text { cells L }{ }^{-1}\right)\end{array}$ & $\begin{array}{l}\text { Months with } \\
\text { maxima }\end{array}$ & $\begin{array}{l}\text { Biovolume } \\
\left(\mu \mathrm{m}^{3} \text { cell }^{-1}\right)\end{array}$ & $\begin{array}{l}\text { Carbon } \\
\left(\text { pg cell }^{-1}\right)\end{array}$ \\
\hline \multicolumn{7}{|l|}{ Order Peridiniales } \\
\hline Diplopsalis lenticula & HT & Jan-Dec & 1,800 & May & 68,147 & 6,909 \\
\hline Heterocapsa cf. niei & MT & Jan-Dec & 135,270 & May & 273 & 75 \\
\hline Heterocapsa $\mathrm{cf}$. rotundata & MT & May-Sep & 5,700 & Jun & 1,077 & 231 \\
\hline Protoperidinium bipes & HT & Jan-Dec & 2,360 & May & 7,079 & 1,081 \\
\hline Protoperidinium brevipes & HT & Feb-Dec & 1,080 & Apr & 17,106 & 2,227 \\
\hline Protoperidinium cf. claudicans & HT & Apr-Oct & 120 & Oct & 88,488 & 8,557 \\
\hline Protoperidinium cf. conicum & HT & Apr-Dec & 240 & May/Aug & 82,966 & 8,117 \\
\hline Protoperidinium $\mathrm{cf}$. divergens & HT & Jan-??? & 20 & Jan & 88,804 & 8,582 \\
\hline Protoperidinium cf. leonis & HT & Mar-Oct & 1,720 & Jul & 50,915 & 5,442 \\
\hline Protoperidinium cf. minutum group & HT & May-Dec & 1,920 & Jul & 20,206 & 2,553 \\
\hline Protoperidinium cf. obtusum & HT & Jun-Oct & 200 & Aug & 146,909 & 12,961 \\
\hline Protoperidinium cf. pyriforme group & HT & Mar-Jan & 4,520 & May & 16,085 & 2,118 \\
\hline Protoperidinium $\mathrm{cf}$. subinerme & HT & May-Dec & 220 & May & 121,344 & 11,082 \\
\hline Protoperidinium denticulatum & HT & Mar-Nov & 80 & Jun/Sep & 40,351 & 4,498 \\
\hline Protoperidinium depressum & HT & May-Nov & 80 & Jul & 513,443 & 36,117 \\
\hline Protoperidinium excentricum & HT & Apr-Dec & 40 & Apr/Sep & 49,635 & 5,329 \\
\hline Protoperidinium ovatum & HT & Mar-Oct & 280 & Apr & 134,256 & 12,039 \\
\hline Protoperidinium pellucidum & HT & Mar-Jun & 3,560 & May & 68,392 & 6,929 \\
\hline Protoperidinium pentagonum & HT & May-Dec & 40 & May/Jul/Aug & 700,146 & 46,561 \\
\hline Protoperidinium sp. $20-30 \mu \mathrm{m}$ & HT & Jan-Dec & 1,120 & Sep & 8,567 & 1,264 \\
\hline Protoperidinium sp. $30-40 \mu \mathrm{m}$ & HT & Mar-Aug & 560 & May/Aug & 31,686 & 3,690 \\
\hline Protoperidinium thorianum & HT & Mar-Nov & 220 & Mar & 119,459 & 10,941 \\
\hline Pyrophacus horologicum & MT & Jun-Sep & 80 & Sep & 56,845 & 5,955 \\
\hline Scrippsiella/Pentapharsodinium sp. & MT & Jan-Dec & 74,965 & Aug & 11,680 & 1,630 \\
\hline \multicolumn{7}{|l|}{ Order Gymnodiniales } \\
\hline Akashiwo sanguinea & MT & Apr-Dec & 56,480 & Oct & 50,539 & 5,409 \\
\hline Amphidinium crassum & HT & Apr-Jan & 1,460 & Jun & 5,089 & 825 \\
\hline Amphidinium cf. sphenoides & HT & Aug-Feb & 60 & Aug/Dec & 3,215 & 566 \\
\hline Lepidodinium chlorophorum & MT & Jul-Jan & 483,402 & Aug & 3,823 & 653 \\
\hline Gymnodinium sp. $20 \mu \mathrm{m}$ & MT & Jan-Dec & 1,600 & Nov & 2,817 & 508 \\
\hline Gymnodinium sp. $30 \mu \mathrm{m}$ & MT & Jan-Dec & 1,280 & Jul & 13,343 & 1,817 \\
\hline Gymnodinium sp. $50 \mu \mathrm{m}$ & MT & Mar-??? & 20 & Mar & 43,000 & 4,738 \\
\hline Gyrodinium calyptoglyphe & HT & Jan-Dec & 180 & Jan & 11,875 & 1,652 \\
\hline Gyrodinium/Gymnodinium spp. $<15 \mu \mathrm{m}$ & HT & Jan-Dec & 74,965 & Oct & 733 & 169 \\
\hline Gyrodinium sp. $20 \mu \mathrm{m}$ & HT & Jul-Apr & 34,578 & Oct & 1,014 & 220 \\
\hline Gyrodinium sp. $20-30 \mu \mathrm{m}$ & HT & Jan-Dec & 5,160 & Oct & 3,896 & 663 \\
\hline Gyrodinium sp. $30-50 \mu \mathrm{m}$ & HT & Jan-Dec & 7,280 & Jul & 8,774 & 1,289 \\
\hline Gyrodinium sp. 50-75 $\mu \mathrm{m}$ & HT & Feb-Dec & 5,560 & Apr & 28,746 & 3,407 \\
\hline Gyrodinium sp. $75-100 \mu \mathrm{m}$ & HT & Feb-Dec & 1,320 & Apr & 54,520 & 5,755 \\
\hline Gyrodinium sp. $100-150 \mu \mathrm{m}$ & HT & Mar- Nov & 2,080 & May & 178,637 & 15,212 \\
\hline Katodinium sp. $<15 \mu \mathrm{m}$ & HT & Jan-Dec & 30,705 & Aug & 472 & 118 \\
\hline Katodinium sp. $20 \mu \mathrm{m}$ & HT & Jan-Dec & 5,256 & Jun & 1,417 & 290 \\
\hline Katodinium glaucum & HT & Jan-Dec & 10,400 & Aug & 5,599 & 892 \\
\hline Nematodinium sp. & HT & Jun-Dec & 560 & Nov & 16,428 & 2,155 \\
\hline Polykrikos kofoidii & HT & May-Nov & 940 & Oct & 126,028 & 11,431 \\
\hline Torodinium robustum $35 \mu \mathrm{m}$ & MT & Jan-Dec & 1,440 & Apr & 5,750 & 912 \\
\hline
\end{tabular}


Table 1 continued

\begin{tabular}{|c|c|c|c|c|c|c|}
\hline Dinoflagellates & $\begin{array}{l}\text { Assigned } \\
\text { trophy }\end{array}$ & $\begin{array}{l}\text { Observed } \\
\text { seasonality }\end{array}$ & $\begin{array}{l}\text { Maximum } \\
\left(\text { cells L }{ }^{-1}\right)\end{array}$ & $\begin{array}{l}\text { Months with } \\
\text { maxima }\end{array}$ & $\begin{array}{l}\text { Biovolume } \\
\left(\mu \mathrm{m}^{3} \text { cell }^{-1}\right)\end{array}$ & $\begin{array}{l}\text { Carbon } \\
\left(\text { pg cell }^{-1}\right)\end{array}$ \\
\hline Torodinium robustum $60 \mu \mathrm{m}$ & MT & Jan-Dec & 620 & Aug & 16,342 & 2,145 \\
\hline Warnowia sp. & HT & Oct-Jul & 4,560 & May & 10,799 & 1,528 \\
\hline \multicolumn{7}{|l|}{ Order Gonyaulacales } \\
\hline Ceratium furca & MT & Apr-Jan & 300 & May & 34,730 & 3,978 \\
\hline Ceratium fusus & MT & Jan-Dec & 4,520 & Jul & 20,830 & 2,617 \\
\hline Ceratium horridum & MT & Sep-Jun & 340 & Jan & 65,256 & 6,668 \\
\hline Ceratium lineatum & MT & May-Jan & 5,740 & Jun & 30,563 & 3,583 \\
\hline Gonyaulax cf. spinifera & MT & Jun-Aug & 280 & Aug & 22,327 & 2,770 \\
\hline Peridiniella $\mathrm{cf}$. danica & HT & Apr-Jan & 12,172 & Jun & 1,424 & 291 \\
\hline \multicolumn{7}{|l|}{ Order Prorocentrales } \\
\hline Mesoporos sp. & MT & Jan-Dec & 1,360 & Aug & 3,709 & 637 \\
\hline Prorocentrum balticum & MT & Jan-Dec & 32,780 & Jun & 655 & 154 \\
\hline Prorocentrum micans & MT & Jan-Dec & 3,960 & Jul & 10,414 & 1,483 \\
\hline Prorocentrum triestinum & MT & Jul-Dec & 56,432 & Aug & 2,106 & 401 \\
\hline \multicolumn{7}{|l|}{ Order Noctilucales } \\
\hline Noctiluca scintillans & HT & May-Jan & 820 & Jun & $166,670,105$ & 308,671 \\
\hline Spatulodinium pseudonoctiluca & HT & Apr-Aug & 20 & Apr/Aug & 960,073 & 60,300 \\
\hline \multicolumn{7}{|l|}{ Order Dinophysiales } \\
\hline Dinophysis sp. & MT & Jan-Dec & 1,560 & Aug & 20,320 & 2,564 \\
\hline
\end{tabular}

ciliate groups were of negligible importance from a biomass perspective $(0.4-1 \%)$.

Ciliates showed a different succession pattern when compared to dinoflagellates. Although they also generally followed the development of chlorophyll $a$ in spring, they responded with an earlier and steeper increase to enhanced food availability (Fig. 3). Maxima were again found earlier in the year (March-early June) compared to dinoflagellates and mainly comprised Strombidiida (Laboea strobila, $S$. capitatum, S. cf. acutum, S. cf. emergens, S. cf. epidemum, S. cf. lynii and S. cf. tressum) and Cyclotrichium spp. As for the dinoflagellates and chlorophyll $a$, we sometimes detected high fluctuations in ciliate biomass (i.e. spring 2007/2009) due to variations in water bodies that did not reflect natural growth or mortality rates.

During the summer months, heterotrophic ciliate biomass often fluctuated synchronised with chlorophyll a concentration (summer 2007); during autumn (October 2007, September 2008), ciliate peaks followed those of autotrophic biomass. Towards winter, ciliate biomass also decreased in parallel with declining chlorophyll $a$ concentrations. Interestingly, the first ciliate peak in 2007/2008 even occurred before the peak of the phytoplankton spring bloom.

Apart from three sampling dates during winter, the mixotrophic species $M$. rubra was present throughout the whole year usually in concentrations of up to $25 \mu \mathrm{gC} \mathrm{L}^{-1}$.
It gained in importance during late spring and summer where it sometimes surpassed the biomass of the remaining ciliates. Maximum concentrations of this ciliate were found in spring 2007 ( $25 \mu \mathrm{gC} \mathrm{L}^{-1}$, small cells) and in June 2009 (97 $\mu \mathrm{gC} \mathrm{L} \mathrm{L}^{-1}$, large cells).

Status of long-term monitoring on dinoflagellates and ciliates at Helgoland Roads

The revision and quality analysis of the long-term data set on plankton by Wiltshire and Dürselen (2004) showed that quality control was very arduous and is an ongoing process. Reasons which hampered the evaluation were both methodological in nature (e.g. fixation procedures or new microscope optics) and due to the frequent change of analysts during certain periods of the time series. The personal element involved in the recognition of microplankton species can never be eliminated completely, and especially for the dinoflagellates, it became evident that there was a large difference in the taxonomic knowledge between the ten different analysts. The revision also revealed that several taxa that have been recorded continuously since 1962 can be used without any restriction (12 diatom and 6 dinoflagellate taxa) and that others can be used with only minor restrictions (7 diatom and 2 dinoflagellate taxa) (for detailed account see Wiltshire and Dürselen 2004). 
Table 2 Ciliate taxa and their seasonality as well as maximum cell concentrations, the mean biovolume and mean carbon content of each taxon as recorded during the 2.5 years of microzooplankton monitoring

\begin{tabular}{|c|c|c|c|c|c|c|}
\hline Ciliates & $\begin{array}{l}\text { Assigned } \\
\text { trophy }\end{array}$ & $\begin{array}{l}\text { Observed } \\
\text { seasonality }\end{array}$ & $\begin{array}{l}\text { Maximum } \\
\left(\text { cells L }{ }^{-1}\right)\end{array}$ & $\begin{array}{l}\text { Months with } \\
\text { maxima }\end{array}$ & $\begin{array}{l}\text { Biovolume } \\
\left(\mu \mathrm{m}^{3} \text { cell }^{-1}\right)\end{array}$ & $\begin{array}{l}\text { Carbon } \\
\left(\text { pg cell }^{-1}\right)\end{array}$ \\
\hline \multicolumn{7}{|l|}{ Order Strombidiida } \\
\hline Cyrtostrombidium sp. $70 \mu \mathrm{m}$ & $\mathrm{HT}$ & Jan-Dec & 480 & Apr & 9,845 & 1,871 \\
\hline Cyrtostrombidium sp. $160 \mu \mathrm{m}$ & HT & Apr-Jan & 80 & Oct & 170,893 & 32,470 \\
\hline Laboea strobila & HT & Feb-Nov & 2,240 & Mar & 91,865 & 17,454 \\
\hline Strombidium capitatum & HT & Feb-Oct & 1,120 & Apr & 99,785 & 18,959 \\
\hline Strombidium cf. acutum & HT & Jan-Dec & 3,020 & Apr & 43,563 & 8,277 \\
\hline Strombidium cf. conicum & HT & Mar-Sep & 360 & May & 24,877 & 4,727 \\
\hline Strombidium cf. emergens & HT & Jan-Aug & 1,080 & Apr & 35,923 & 6,825 \\
\hline Strombidium cf. epidemum $30 \mu \mathrm{m}$ & HT & Jan-Dec & 24,840 & Apr & 6,465 & 1,228 \\
\hline Strombidium cf. epidemum $35 \mu \mathrm{m}$ & HT & Jan-Oct & 1,320 & Aug & 8,862 & 1,684 \\
\hline Strombidium cf. lynii & HT & Jan-Dec & 400 & Jan & 36,968 & 7,024 \\
\hline Strombidium cf. tressum & HT & Mar-Sep & 2,640 & Apr & 9,694 & 1,842 \\
\hline Strombidium sp. $20 \mu \mathrm{m}$ & HT & Jan-Dec & 1,640 & May & 4,977 & 946 \\
\hline Strombidium sp. $25 \mu \mathrm{m}$ & HT & Feb-Sep & 1,680 & Jul & 2,463 & 468 \\
\hline Strombidium sp. A $30 \mu \mathrm{m}$ & HT & Jan-Dec & 540 & Apr & 5,893 & 1,120 \\
\hline Strombidium sp. B $30 \mu \mathrm{m}$ & HT & Sep-May & 200 & May/Oct & 4,157 & 790 \\
\hline Strombidium sp. $35 \mu \mathrm{m}$ & HT & Jan-Dec & 580 & Aug & 8,244 & 1,566 \\
\hline Strombidium sp. $40 \mu \mathrm{m}$ & HT & Jan-Dec & 2,480 & May & 7,296 & 1,386 \\
\hline Strombidium sp. $50 \mu \mathrm{m}$ & HT & Jan-Sep & 100 & Aug/Sep & 9,390 & 1,784 \\
\hline Strombidium sp. $60 \mu \mathrm{m}$ & HT & Feb-Dec & 1,200 & Jul & 19,294 & 3,666 \\
\hline Strombidium sp. $100 \mu \mathrm{m}$ & HT & Jan-Dec & 120 & Apr & 105,853 & 20,112 \\
\hline Tontonia gracillima & HT & Feb-Dec & 280 & Apr/May/Jun & 25,089 & 4,767 \\
\hline \multicolumn{7}{|l|}{ Order Choreotrichida } \\
\hline Leegaardiella cf. ovalis & HT & Jan-Dec & 380 & Feb & 5,594 & 1,063 \\
\hline Leegaardiella cf. sol & HT & Jan-Dec & 780 & Apr & 22,301 & 4,237 \\
\hline Lohmanniella oviformis & HT & Nov-Jul & 2,260 & Mar & 4,725 & 898 \\
\hline Rimostrombidium sp. & HT & Feb-Sep & 320 & Apr & 100,779 & 19,148 \\
\hline Strobilidium cf. neptunii & HT & Feb-Nov & 160 & May/Jun & 54,726 & 10,398 \\
\hline Strobilidium cf. sphaericum & HT & Jul-??? & 40 & Jul & 74,475 & 14,150 \\
\hline Strobilidium cf. spiralis & HT & Apr-??? & 40 & Apr & 18,578 & 3,530 \\
\hline Strobilidium sp. $15 \mu \mathrm{m}$ & HT & May-Nov & 10,160 & Aug & 1,061 & 202 \\
\hline Strobilidium sp. $45 \mu \mathrm{m}$ & HT & May-Aug & 400 & Aug & 38,186 & 7,255 \\
\hline Strombidinopsis sp. $90 \mu \mathrm{m}$ & HT & Dec-Apr & 40 & Dec/Jan/Mar & 94,898 & 18,031 \\
\hline Strombidinopsis sp. $120 \mu \mathrm{m}$ & HT & Jul-Oct & 120 & Aug & 103,546 & 19,674 \\
\hline \multicolumn{7}{|l|}{ Order Tintinnida } \\
\hline Eutintinnus sp. $30 \mu \mathrm{m}$ & HT & Jun-Oct & 240 & Oct & 4,169 & 792 \\
\hline Eutintinnus sp. $60 \mu \mathrm{m}$ & HT & Aug-??? & 120 & Aug & 32,695 & 6,212 \\
\hline Favella ehrenbergii & HT & Jul-Oct & 280 & Sep & 100,917 & 19,174 \\
\hline Salpingella sp. & HT & Oct-Jan & 260 & Nov & 1,953 & 371 \\
\hline Stenosemella sp. & HT & Nov-Jun & 6,440 & May & 10,722 & 2,037 \\
\hline Tintinnid sp. $30 \mu \mathrm{m}$ & HT & Jan-Dec & 1,800 & Jun & 1,496 & 284 \\
\hline Tintinnid sp. $70 \mu \mathrm{m}$ & HT & Sep-??? & 40 & Sep & 58,219 & 11,062 \\
\hline Tintinnidium cf. balechi & HT & Jan-Dec & 1,360 & Jan & 4,114 & 782 \\
\hline Tintinnopsis cf. radix & $\mathrm{HT}$ & Aug-Oct & 80 & Oct & 27,489 & 5,223 \\
\hline Tintinnopsis sp. & HT & Mar-Jan & 660 & Nov & 4,279 & 813 \\
\hline \multicolumn{7}{|l|}{ Order Cyclotrichiida } \\
\hline Askenasia regina & HT & Aug-Oct & 380 & Oct & 172,422 & 32,760 \\
\hline
\end{tabular}


Table 2 continued

\begin{tabular}{|c|c|c|c|c|c|c|}
\hline Ciliates & $\begin{array}{l}\text { Assigned } \\
\text { trophy }\end{array}$ & $\begin{array}{l}\text { Observed } \\
\text { seasonality }\end{array}$ & $\begin{array}{l}\text { Maximum } \\
\left(\text { cells L }^{-1}\right)\end{array}$ & $\begin{array}{l}\text { Months with } \\
\text { maxima }\end{array}$ & $\begin{array}{l}\text { Biovolume } \\
\left(\mu \mathrm{m}^{3} \text { cell }^{-1}\right)\end{array}$ & $\begin{array}{l}\text { Carbon } \\
\left(\text { pg cell }^{-1}\right)\end{array}$ \\
\hline Askenasia sp. & HT & Apr-Sep & 60 & Jun & 269,492 & 51,203 \\
\hline Mesodinium pulex & HT & Jan-Dec & 1,600 & Aug & 2,258 & 429 \\
\hline Mesodinium sp. $20 \mu \mathrm{m}$ & HT & May-Mar & 680 & Aug & 4,102 & 779 \\
\hline Mesodinium sp. $45 \mu \mathrm{m}$ & HT & May-Sep & 160 & Jul/Aug & 50,965 & 9,683 \\
\hline Myrionecta rubra $15 \mu \mathrm{m}$ & MT & Jan-Dec & 24,960 & Apr & 2,356 & 448 \\
\hline Myrionecta rubra $35 \mu \mathrm{m}$ & MT & Jan-Dec & 23,560 & Jun & 21,637 & 4,111 \\
\hline \multicolumn{7}{|l|}{ Order Haptorida } \\
\hline Cyclotrichium sp. & HT & Mar-Oct & 380 & Apr & 595,288 & 113,105 \\
\hline Didinium gargantua & HT & Jun-Aug & 20 & Jun/Aug & 68,770 & 13,066 \\
\hline Spathidium sp. & HT & Mar-Nov & 500 & Jun & 5,864 & 1,114 \\
\hline \multicolumn{7}{|l|}{ Order Prorodontida } \\
\hline Balanion comatum $10-15 \mu \mathrm{m}$ & HT & May-Jan & 4,426 & Jun & 443 & 84 \\
\hline Balanion comatum $15-25 \mu \mathrm{m}$ & HT & Jan-Nov & 1,880 & Jun & 2,356 & 448 \\
\hline Tiarina fusus & HT & Jun-Dec & 1,520 & Aug & 20,425 & 3,881 \\
\hline \multicolumn{7}{|l|}{ Order Euplotida } \\
\hline Euplotes sp. & HT & Jan-Dec & 1,040 & Aug & 7,561 & 1,437 \\
\hline \multicolumn{7}{|l|}{ Subclass Scuticociliatia } \\
\hline Scuticociliates $10-30 \mu \mathrm{m}$ & HT & Jan-Dec & 2,240 & Apr & 1,595 & 303 \\
\hline Scuticociliates $30-50 \mu \mathrm{m}$ & HT & Apr-Jan & 1,320 & Aug & 17,641 & 3,352 \\
\hline \multicolumn{7}{|l|}{ Sessile ciliates } \\
\hline Acineta sp. & HT & Apr-Aug & 60 & Aug & 35,298 & 6,707 \\
\hline Vorticella sp. & HT & Jan-Dec & 620 & May & 6,729 & 1,278 \\
\hline \multicolumn{7}{|l|}{ Miscellaneous } \\
\hline Strombidium/Strobilidium spp. $<10 \mu \mathrm{m}$ & HT & Jan-Dec & 3,043 & Jun & 347 & 66 \\
\hline Strombidium/Strobilidium spp. $<15 \mu \mathrm{m}$ & HT & Jan-Dec & 19,360 & Jun & 805 & 153 \\
\hline
\end{tabular}

Fig. 1 Proportion of different dinoflagellate (left panel) and ciliate groups (right panel) during the 2.5 years of monitoring based on their carbon biomass contribution. MT mixotrophic, $H T$ heterotrophic
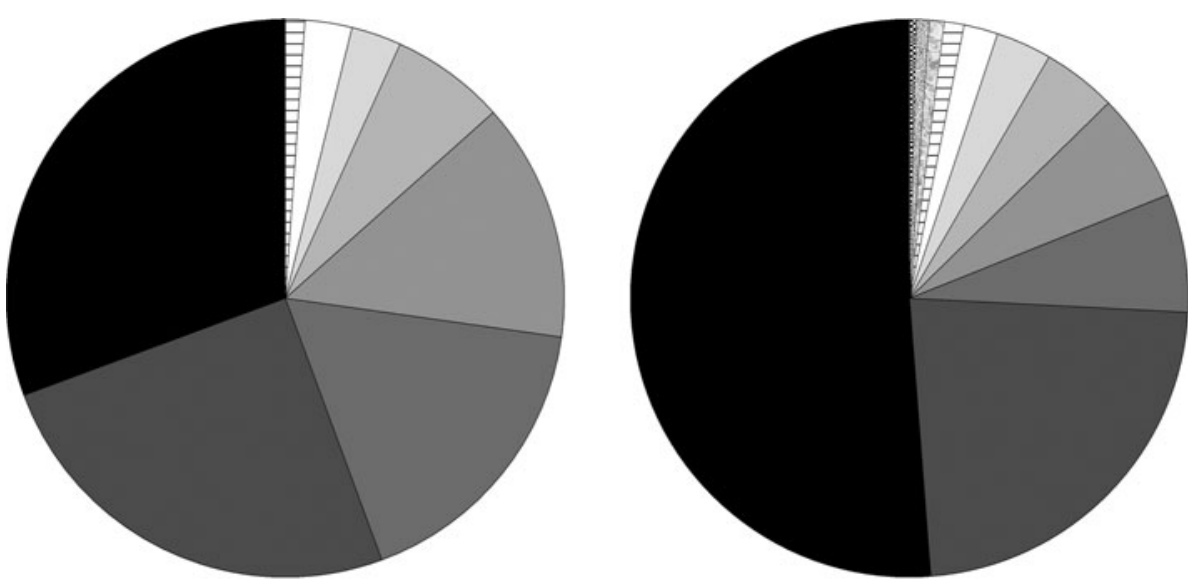

Gymnodiniales (MT)

Noctilucales

Gymnodiniales (HT)

Peridiniales (MT)

Peridiniales (HT)

Prorocentrales

Gonyaulacales

Dinophysiales
Strombidiida

Myrionecta rubra

Choreotrichida

Haptorida

Cyclotrichiida

Tintinnida

Prorodontida

Scuticociliata

Euplotida

Miscellaneous

$\approx \times m \times$ Sessile ciliates 


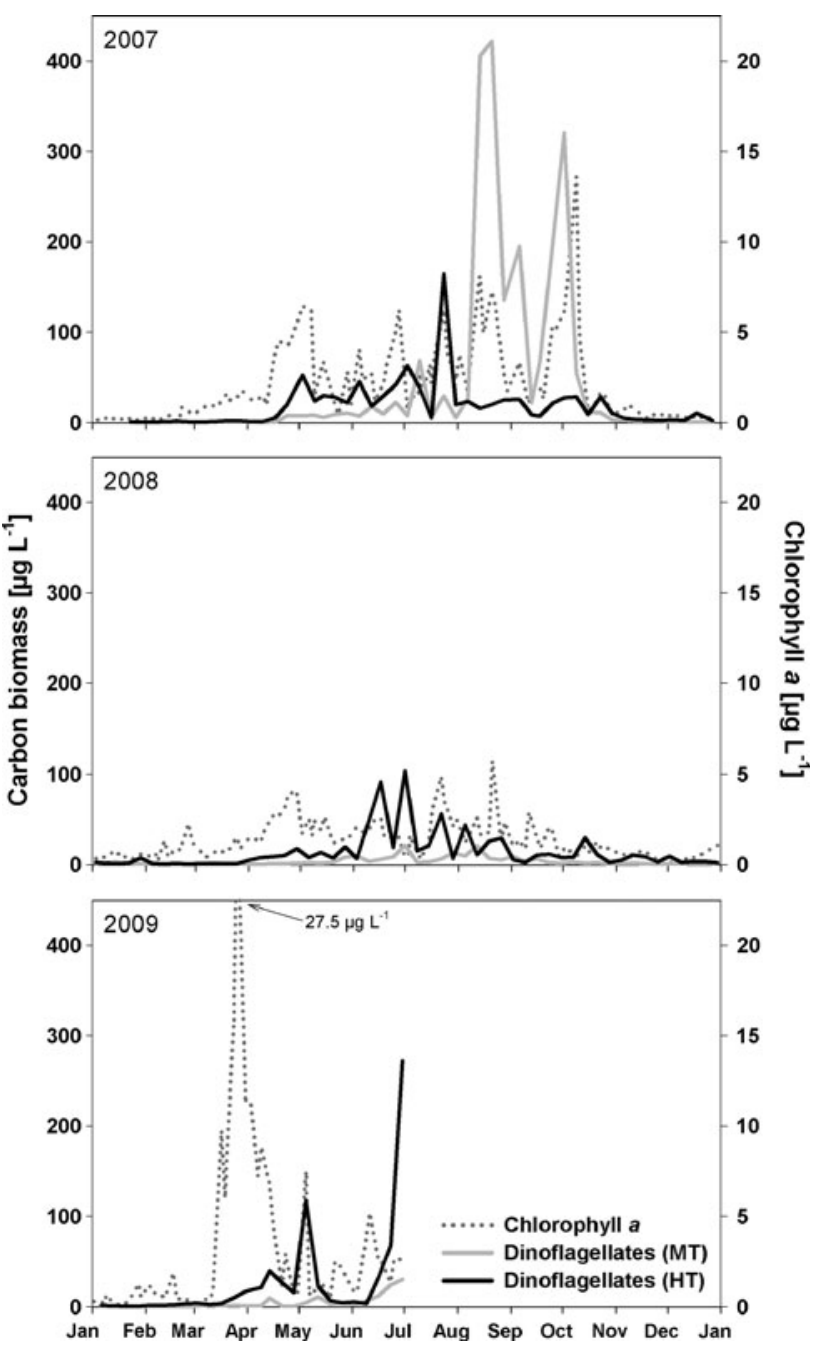

Fig. 2 Carbon biomass $\left(\mu \mathrm{gC} \mathrm{L}{ }^{-1}\right)$ of mixotrophic (MT) and heterotrophic (HT) dinoflagellates during the time of a 2.5-year weekly monitoring programme at Helgoland Roads in comparison with chlorophyll $a$ concentration $\left(\mu \mathrm{g} \mathrm{L}^{-1}\right)$ measured on a work-daily basis via in situ fluorescence as a regular parameter of the long-term series

Here, we focus on the long-term data of dinoflagellates and ciliates as these two groups were the major interest of this study. A new revision of the long-term data in 2008 showed that only a restricted number of 9 dinoflagellate taxa were recorded continuously since the start of longterm monitoring and that these can be used without limitation (M. Scharfe and S. Peters, unpublished). These comprised different Ceratium species (C. furca, C. fusus, C. horridum, C. lineatum, C. tripos), Prorocentrum micans, the groups Gyrodinium spp. and Protoperidinium spp. as well as the species Noctiluca scintillans. Compared with the microzooplankton monitoring reported here, the 9 taxa of the long-term data set that can be used without limitation
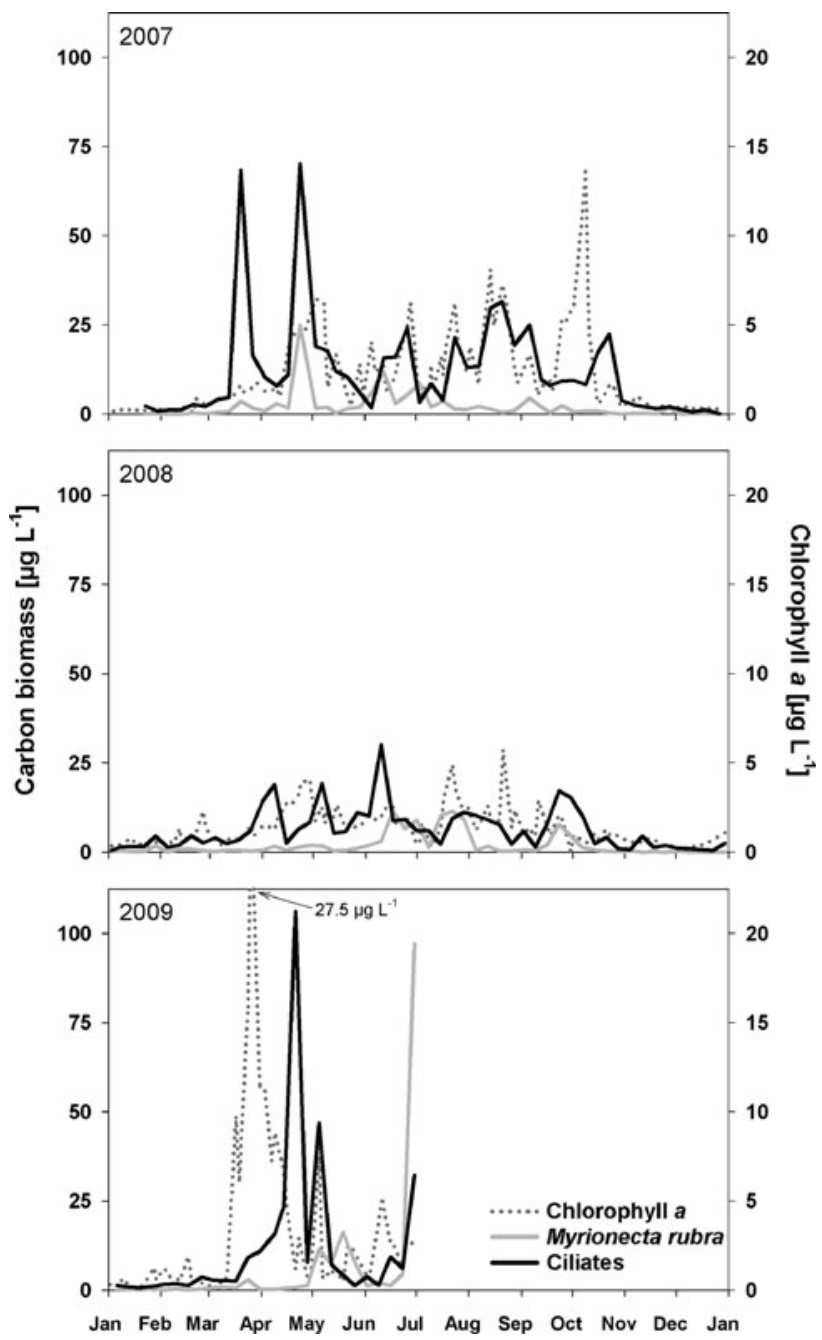

Fig. 3 Carbon biomass $\left(\mu \mathrm{gC} \mathrm{L} \mathrm{L}^{-1}\right)$ of the ciliate Myrionecta rubra and the sum of the remaining ciliates during the time of a 2.5 -year weekly monitoring programme at Helgoland Roads in comparison with chlorophyll $a$ concentration ( $\mu \mathrm{g} \mathrm{L}^{-1}$ ) measured on a work-daily basis via in situ fluorescence as a regular parameter of the long-term series

represented on average only $61 \%$ (3-94\%) of the dinoflagellate biomass recorded during the 2.5 years.

Ciliates represented $3-96 \%$ of the total microzooplankton biomass recorded during the 2.5 years of monitoring. Their mean biomass contribution of $36 \%$ shows the importance of this microzooplankton group. Nevertheless, no ciliate species was recorded before 1999 when the longterm plankton monitoring started to include Myrionecta rubra. In the year 2007 Laboea strobila and 2008 Mesodinium pulex were additionally counted in the samples. However, these three ciliate taxa represented on average only $30 \%(0-86 \%)$ of the ciliate biomass recorded during the microzooplankton monitoring at Helgoland Roads. 
Comparison of the two monitoring programmes

Due to their important contribution to planktonic biomass when concerning our data (Fig. 1) and due to the availability of long-term quality-checked cell concentration data on both species, we chose the dinoflagellate Noctiluca scintillans and the ciliate Myrionecta rubra for comparison of the 2.5-year data set with the data of the long-term series. As the long-term data series provided only rough carbon biomass values for those two species (Wiltshire and Dürselen 2004), we used cell numbers $\left[\mathrm{n} \mathrm{L}^{-1}\right]$ for comparisons.

Noctiluca scintillans (Fig. 4a) has continuously been recorded in the long-term data since 1962. It is the largest heterotrophic dinoflagellate species (usually $>500 \mu \mathrm{m}$ ) at Helgoland Roads. This species cannot be overlooked, and its characteristic appearance prevents confusion with other dinoflagellate species. $N$. scintillans usually occurred in higher densities from May to September with only rare observations in the other months of the year. One exception was the year 1965 where it was recorded only on 2 days at very low densities. Maxima were found in summer (JuneAugust) reaching concentrations of up to 22.500 cells $\mathrm{L}^{-1}$.

Myrionecta rubra (Fig. 4b) has been recorded since 1999. This bloom-forming ciliate can be found in different size classes (Montagnes et al. 2008), and at Helgoland Roads, the size classes $\sim 15 \mu \mathrm{m}$ and $\sim 35 \mu \mathrm{m}$ were recorded during the microzooplankton monitoring. No differentiation in size classes was made in the long-term monitoring. It showed an all year-round occurrence at Helgoland Roads with minimal cell concentrations in wintertime. Frequently, two distinct maxima were found
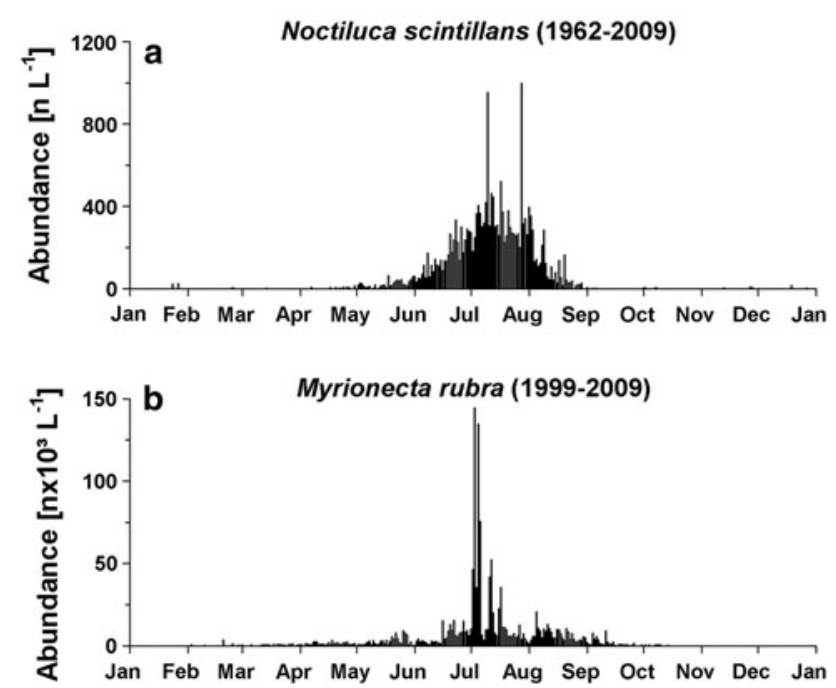

Fig. 4 Mean daily cell concentration of (a) the dinoflagellate Noctiluca scintillans $\left(\mathrm{n} \mathrm{L} \mathrm{L}^{-1}\right)$ during the years 1962-2009 and (b) the ciliate Myrionecta rubra $\left(\mathrm{n} \times 10^{3} \mathrm{~L}^{-1}\right)$ during the years 1999-2009 of long-term monitoring at Helgoland Roads within the year: a lower spring maximum and a pronounced summer maximum where cell concentration partly rose up to over $1.1 \times 10^{6}$ cells $\mathrm{L}^{-1}$. In the recent years (2007-2009), M. rubra concentration was generally lower than in previous years. Interestingly, when looking at the data of the first 2 years in which this species has been counted, it became obvious that $M$. rubra cells were only recorded during a narrow window in the summer months, while in the following years it occurred year-round. This pattern is due to the two size classes of M. rubra. The smaller size class is more abundant in winter and spring than the bigger one; thus, it can easily be overlooked especially by an inexperienced analyst who has just started to count M. rubra. This was the case in the year 1999 .

The comparison of the data of the weekly microzooplankton monitoring with the data of the work-daily counts (Figs. 5, 6) revealed that despite small differences, the lower resolution in the microzooplankton monitoring could nevertheless describe the seasonal patterns of distribution in both species. Discrepancies between both monitoring programmes were more pronounced in $N$. scintillans (Fig. 5a, b), where especially the maximum values of the years 2007 and 2009 were not reflected in the weekly samples. M. rubra (Fig. 6a, b) concentration from the microzooplankton monitoring mirrored the long-term data quite well. The most obvious outlier was in April 2007 where the microzooplankton monitoring recorded much higher concentrations of the small size class of M. rubra. This was due to methodological differences: besides the different counting frequencies, deviations in the records of both species most probably resulted from differences in counting methodology. While in the long-term monitoring, lower volumes are settled during blooms (usually $25 \mathrm{~mL}$ ) and often tracks are counted for the smaller species (as here for M.rubra), at least half of the sedimentation chamber was counted during the microzooplankton monitoring and $50 \mathrm{~mL}$ were always used for sedimentation. Therefore, patchy settlement in a counting chamber will not have such a great effect as in the long-term monitoring. In conclusion, despite minor differences, data on $N$. scintillans and M. rubra of both monitoring programmes were well-matched and showed that the less frequent microzooplankton monitoring was suited for describing seasonal dynamics of dinoflagellates and ciliates.

Ecological implications of the microzooplankton monitoring data

Our seasonal results for ciliates are comparable to results from monitoring programmes in the North Sea (Brussaard et al. 1995), Baltic Sea (Smetacek 1981; Johansson et al. 2004) and the Gulf of Maine (Montagnes et al. 1988) where distinct spring peaks were also seen. As they can respond 


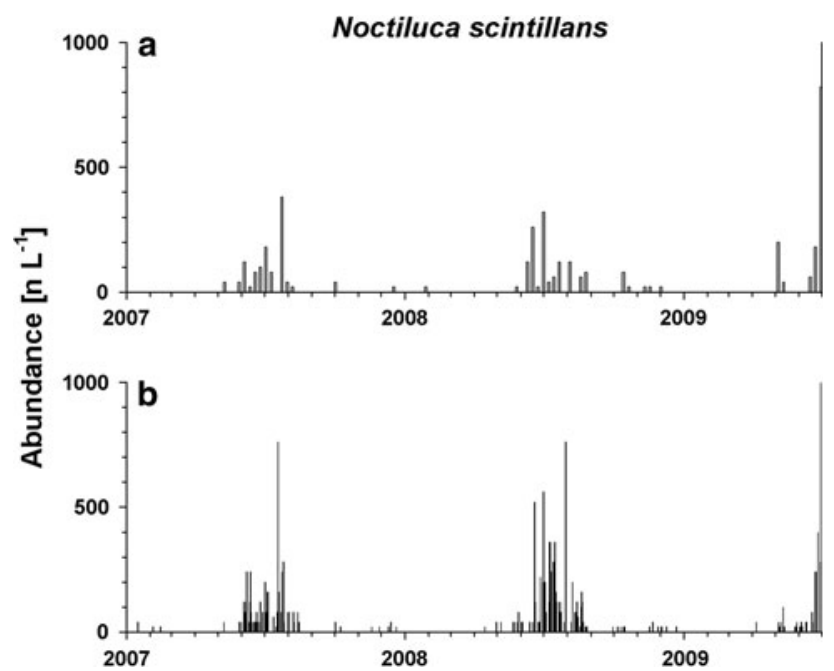

Fig. 5 Comparison of cell concentration data on Noctiluca scintillans (n $\mathrm{L}^{-1}$ ) between the 2.5 years of microzooplankton monitoring (a) and the long-term monitoring (b)

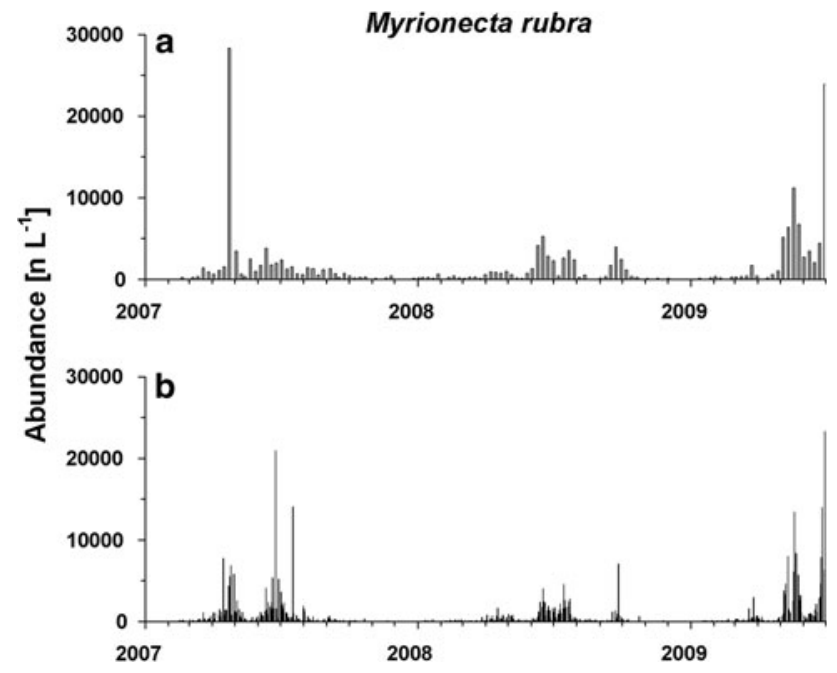

Fig. 6 Comparison of cell concentration data on Myrionecta rubra (n $\mathrm{L}^{-1}$ ) between the 2.5 years of microzooplankton monitoring (a) and the long-term monitoring (b)

more quickly to increasing phytoplankton concentrations, ciliates play a key role during spring (Riegman et al. 1993) and form an earlier peak than dinoflagellates. The majority of ciliates is, with few exceptions (Smetacek 1981; Aberle et al. 2007), restricted to the availability of smaller prey (Jonsson 1986; Tillmann 2004) consisting mainly of flagellates (Kivi and Setälä 1995), and their seasonal co-occurrence can be linked to that fact. Heterotrophic dinoflagellates are generally directly related to the availability of larger phytoplankton prey (Hansen 1991) and often occur at high concentrations during the course of diatom blooms (Sherr and Sherr 2007) especially during spring blooms (Stelfox-Widdicombe et al. 2004). Hansen
(1991) reported a close relationship between dinoflagellate concentration and prey availability, and this was also shown by our results.

Microzooplankton can be both prey and competitor for mesozooplankton. At Helgoland Roads, small calanoid copepods can be regarded as direct competitors of ciliates and dinoflagellates for phytoplankton food. Their concentration ranges between 2 and 10 individuals $\mathrm{L}^{-1}$ over the year, with highest values during the summer period (Greve et al. 2004). The mean carbon content (annual mean 2007, $n=45$ ) of the abundant small calanoid copepod Temora longicornis (Greve et al. 2004) was $9.5 \mu \mathrm{g}$ carbon per female (K. L. Schoo, unpublished) at Helgoland Roads. Assuming a maximum carbon content of $10 \mu \mathrm{g}$ per copepod combined with the maximum concentrations given by Greve et al. (2004) would therefore result in a maximum copepod carbon biomass of $100 \mu \mathrm{g} \mathrm{L}^{-1}$ (June/July). This value was surpassed by microzooplankton biomass, especially during the spring bloom. At this time, the combined effects of a faster metabolism and higher productivity (Fenchel and Finlay 1983; Montagnes and Lessard 1999) enables microzooplankton to have an undelayed direct response to increases in prey availability (Johansson et al. 2004; Aberle et al. 2007) when compared to its copepod competitors. Therefore, it is hardly surprising that recent studies have shown that microzooplankton does not only compete with copepods for the same resources (Aberle et al. 2007) but that it may exert a stronger grazing pressure on phytoplankton than copepods (Sherr and Sherr 2007) especially during bloom events. Indeed, the results of microzooplankton grazing experiments conducted during the spring bloom 2009 confirmed the dominant role of microzooplankton as phytoplankton grazers at Helgoland Roads when compared to copepods (Löder 2010).

We found that during the summer months, ciliate biomass was generally lower when compared to dinoflagellate biomass. Only with their decreasing concentrations at the end of summer did ciliate biomass reach the same importance as dinoflagellate biomass again. However, ciliates are the first microzooplankton grazers which react to enhanced food availability in spring when the concentration of small flagellated prey increases at Helgoland. Such an earlier onset of ciliate blooms can be directly linked to their higher growth rates when compared to dinoflagellates (Hansen 1992; Strom and Morello 1998). On the other hand, they are generally more restricted to the availability of particular prey types (Tillmann 2004), especially flagellates, than dinoflagellates (Jeong 1999). Therefore, ciliates can respond more rapidly to enhanced food concentrations than dinoflagellates, but their potential of surviving starvation periods is low (Jackson and Berger 1985) compared to dinoflagellates (Hansen 1992; Menden-Deuer et al. 2005). Consequently, ciliates can be classified as rapid-reaction 
food specialists and dinoflagellates more as generalists with longer response times but greater persistence. This implies rapid responses to increasing food concentrations but also quick declines of ciliate concentration as a direct response to decreasing prey concentration as was especially seen during spring at Helgoland. Ciliate maxima should therefore occur only when their appearance is coupled with the sufficient availability of adequate prey. When food availability increases in spring and ciliate predators achieve reproduction rates equal to those of their prey or even higher (Riegman et al. 1993), the effective grazing of ciliates could sometimes even prevent their preferred prey (e.g. flagellates) from blooming. This is most likely the explanation for the ciliate peaks in spring 2007 and 2008, while simultaneously autotrophic biomass stays more or less constant. These ciliate peaks occurred prior to the chlorophyll $a$ peak of the real phytoplankton spring bloom which consisted mainly of larger diatoms of no prey significance for ciliates (Gifford 1988). Such sizedifferentiated microzooplankton grazing control by ciliates, promoting diatom spring blooms of larger species, has already been reported elsewhere for the North Sea (Riegman et al. 1993; Brussaard et al. 1995).

Another factor potentially influencing abundances of both ciliates and dinoflagellates is predation, for example, by copepods. Microzooplankton contributes substantially to copepod diets and is often positively selected by them (Nejstgaard et al. 1997; Fileman et al. 2007). The capacity of microzooplankton to synthesise highly unsaturated fatty acids and sterols makes them good-quality food for copepods (Klein Breteler et al. 1999; Tang and Taal 2005). Especially when phytoplankton prey is nutrient-limited, rendering it a low-quality food, microzooplankton predators are able to dampen stoichiometric constraints of their prey to a certain extent (Malzahn et al. 2010) and are therefore of better nutritional value for copepods compared to phytoplankton. The selective predation of copepods on microzooplankton during the course of the spring bloom 2009 at Helgoland Roads confirmed the important role of microzooplankton as food source for mesozooplankton as well as the role of copepods as controlling factor for microzooplankton (Löder 2010).

We showed that microzooplankton is an important component of the food web at Helgoland Roads. Due to its temporarily very high biomass occurence and presence throughout the year, it can probably be regarded as the most important phytoplankton grazer group. Microzooplankton is an important food source for higher trophic levels such as copepods at Helgoland. As the routine plankton monitoring at Helgoland Roads has a broader focus on diverse phytoplankton organism groups, it cannot resolve the diversity of microzooplankton. Given its key role in the food web, we recommend the long-term implementation of microzooplankton, especially dinoflagellates and ciliates, into the Helgoland Roads long-term sampling programme. Further multivariate statistical analyses are necessary to evaluate the biotic and abiotic factors that drive microzooplankton composition and abundance patterns. Using the data of the Helgoland Roads long-term series, such analyses will further enhance our in-depth understanding of microzooplankton occurrence in the North Sea.

Acknowledgments This study was part of a PhD thesis within the Food Web Project at the Alfred Wegener Institute for Polar and Marine Research, and we are grateful for the funding. Furthermore, we want to thank the crew of the research vessels Aade for providing samples, Kristine Carstens for her help in the laboratory and Mirco Scharfe for his evaluation of the species lists of the long-term series. Last but not least-we thank the whole team of the AWI Food Web Project for their collegiality.

\section{References}

Aberle N, Lengfellner K, Sommer U (2007) Spring bloom succession, grazing impact and herbivore selectivity of ciliate communities in response to winter warming. Oecologia 150(4):668-681

Beran A, Guardiani B, Tamberlich F, Kamburska L, Fonda Umani S (2003) Carbon content and biovolume of the heterotrophic dinoflagellate Noctiluca scintillans from the Northern Adriatic Sea. Paper presented at the Proceedings of the CESUM-BS 2003, Varna. UNESCO, Paris

Brussaard CPD, Riegman R, Noordeloos AAM, Cadee GC, Witte H, Kop AJ, Nieuwland G, Vanduyl FC, Bak RPM (1995) Effects of grazing, sedimentation and phytoplankton cell lysis on the structure of a coastal pelagic food web. Mar Ecol Prog Ser 123(1-3):259-271

Calbet A, Landry MR (2004) Phytoplankton growth, microzooplankton grazing, and carbon cycling in marine systems. Limnol Oceanogr 49(1):51-57

Capriulo GM, Sherr EB, Sherr BF (1991) Trophic behaviour and related community feeding activities of heterotrophic marine protists. In: Reid PC, Turley CM, Burkill PH (eds) Protozoa and their role in marine processes. Springer, Berlin, pp 219-265

Carey PG (1992) Marine interstitial ciliates: an illustrated key. Chapman \& Hall, London, New York

Dodge JD (1982) Marine dinoflagellates of the British Isles. Her Majesty's Stationery Office, London

Du Yoo Y, Jeong HJ, Kim MS, Kang NS, Song JY, Shin W, Kim KY, Lee K (2009) Feeding by phototrophic red-tide dinoflagellates on the ubiquitous marine diatom Skeletonema costatum. J Eukaryot Microbiol 56(5):413-420

Fenchel T, Finlay BJ (1983) Respiration rates in heterotrophic, freeliving protozoa. Microb Ecol 9(2):99-122

Fileman E, Smith T, Harris R (2007) Grazing by Calanus helgolandicus and Para-Pseudocalanus spp. on phytoplankton and protozooplankton during the spring bloom in the Celtic Sea. J Exp Mar Biol Ecol 348(1-2):70-84

Franke HD, Buchholz F, Wiltshire KH (2004) Ecological long-term research at Helgoland (German Bight, North Sea): retrospect and prospect-an introduction. Helgol Mar Res 58(4):223-229

Gifford DJ (1988) Impact of grazing by microzooplankton in the Northwest Arm of Halifax Harbor, Nova Scotia. Mar Ecol Prog Ser 47(3):249-258 
Greve W, Reiners F, Nast J, Hoffmann S (2004) Helgoland Roads meso- and macrozooplankton time-series 1974 to 2004: lessons from 30 years of single spot, high frequency sampling at the only off-shore island of the North Sea. Helgol Mar Res 58(4):274-288

Hansen PJ (1991) Quantitative importance and trophic role of heterotrophic dinoflagellates in a coastal pelagial food web. Mar Ecol Prog Ser 73(2-3):253-261

Hansen PJ (1992) Prey size selection, feeding rates and growth dynamics of heterotrophic dinoflagellates with special emphasis on Gyrodinium spirale. Mar Biol 114(2):327-334

Hillebrand H, Dürselen C-D, Kirschtel D, Pollingher U, Zohary T (1999) Biovolume calculation for pelagic and benthic microalgae. J Phycol 35(2):403-424

Hoppenrath M (2004) A revised checklist of planktonic diatoms and dinoflagellates from Helgoland (North Sea, German Bight). Helgol Mar Res 58(4):243-251

Hoppenrath M, Elbrächter M, Drebes G (2009) Marine phytoplankton: selected microphytoplankton species from the North Sea around Helgoland and Sylt. Kleine Senckenberg-Reihe 49. E. Schweizerbart'sche Verlagsbuchhandlung (Nägele u. Obermiller), Stuttgart

Jackson KM, Berger J (1985) Survivorship curves of ciliate protozoa under starvation conditions and at low bacterial levels. Protistologica 21(1):17-24

Jeong HJ (1999) The ecological role of heterotrophic dinoflagellates in marine plankton community. $\mathrm{J}$ Eucaryot Microbiol 46:390-396

Johansson M, Gorokhova E, Larsson U (2004) Annual variability in ciliate community structure, potential prey and predators in the open northern Baltic Sea proper. J Plankton Res 26(1):67-80

Jonsson PR (1986) Particle size selection, feeding rates and growth dynamics of marine planktonic oligotrichous ciliates (Ciliophora: Oligotrichina). Mar Ecol Prog Ser 33:265-277

Kahl A (1932) Urtiere oder Protozoa. I Wimpertiere. 3 Spirotricha. In: Dahl F (ed) Die Tierwelt Deutschlands und der angrenzenden Meeresteile, vol 25. Gustav Fischer, Jena

Kivi K, Setälä O (1995) Simultaneous measurement of food particle selection and clearance rates of planktonic oligotrich ciliates (Ciliophora: Oligotrichina). Mar Ecol Prog Ser 119:125-137

Klein Breteler WCM, Schogt N, Baas M, Schouten S, Kraay GW (1999) Trophic upgrading of food quality by protozoans enhancing copepod growth: role of essential lipids. Mar Biol 135(1):191-198

Landry MR, Calbet A (2004) Microzooplankton production in the oceans. ICES J Mar Sci 61(4):501-507

Löder MGJ (2010) The role of heterotrophic dinoflagellate and ciliate grazers in the food web at Helgoland Roads, North Sea. Doctoral thesis, Jacobs University Bremen

Lund JWG, Kipling C, Cren ED (1958) The inverted microscope method of estimating algal numbers and the statistical basis of estimations by counting. Hydrobiologia 11(2):143-170

Malzahn AM, Hantzsche F, Schoo KL, Boersma M, Aberle N (2010) Differential effects of nutrient-limited primary production on primary, secondary or tertiary consumers. Oecologia 162(1): $35-48$

Menden-Deuer S, Lessard EJ (2000) Carbon to volume relationships for dinoflagellates, diatoms, and other protist plankton. Limnol Oceanogr 45(3):569-579

Menden-Deuer S, Lessard EJ, Satterberg J, Grünbaum D (2005) Growth rates and starvation survival of three species of the pallium-feeding, thecate dinoflagellate genus Protoperidinium. Aquat Microb Ecol 41(2):145-152

Montagnes DJS (2003) Planctonic ciliate project internet homepage: http://www.liv.ac.uk/ciliate/intro.htm, date: 19.12.2007, time: 13:56
Montagnes DJS, Lessard EJ (1999) Population dynamics of the marine planktonic ciliate Strombidinopsis multiauris: its potential to control phytoplankton blooms. Aquat Microb Ecol 20(2):167-181

Montagnes DJS, Lynn DH, Roff JC, Taylor WD (1988) The annual cycle of heterotrophic planktonic ciliates in the waters surrounding the Isles of Shoals, Gulf of Maine: an assessment of their trophic role. Mar Biol 99:21-30

Montagnes DJS, Allen J, Brown L, Bulit C, Davidson R, Diaz-Avalos C, Fielding S, Heath M, Holliday NP, Rasmussen J, Sanders R, Waniek JJ, Wilson D (2008) Factors controlling the abundance and size distribution of the phototrophic ciliate Myrionecta rubra in open waters of the North Atlantic. J Eukaryot Microbiol 55(5):457-465

Nejstgaard JC, Gismervik I, Solberg PT (1997) Feeding and reproduction by Calanus finmarchicus, and microzooplankton grazing during mesocosm blooms of diatoms and the coccolithophore Emiliania huxleyi. Mar Ecol Prog Ser 147(1-3):197217

Park JS, Myung G, Kim HS, Cho BC, Yih W (2007) Growth responses of the marine photosynthetic ciliate Myrionecta rubra to different cryptomonad strains. Aquat Microbial Ecol 48:83-90

Putt M, Stoecker DK (1989) An experimentally determined carbon:volume ratio for marine "oligotrichous" ciliates from estuarine and coastal waters. Limnol Oceanogr 34(9):1097-1103

Riegman R, Kuipers BR, Noordeloos AAM, Witte HJ (1993) Sizedifferential control of phytoplankton and the structure of plankton communities. Neth J Sea Res 31(3):255-265

Schlüter MH, Merico A, Wiltshire KH, Greve W, von Storch $\mathrm{H}$ (2008) A statistical analysis of climate variability and ecosystem response in the German Bight. Ocean Dyn 58(3-4):169-186

Sherr EB, Sherr BF (2002) Significance of predation by protists in aquatic microbial food webs. Antonie Van Leeuwenhoek Int $\mathbf{J}$ Gen Mol Microbiol 81(1-4):293-308

Sherr EB, Sherr BF (2007) Heterotrophic dinoflagellates: a significant component of microzooplankton biomass and major grazers of diatoms in the sea. Mar Ecol Prog Ser 352:187-197

Smetacek V (1981) The annual cycle of proto-zooplankton in the Kiel Bight. Mar Biol 63(1):1-11

Stelfox-Widdicombe CE, Archer SD, Burkill PH, Stefels J (2004) Microzooplankton grazing in Phaeocystis and diatom-dominated waters in the southern North Sea in spring. J Sea Res 51:37-51

Stoecker DK, Gifford DJ, Putt M (1994) Preservation of marine planktonic ciliates-losses and cell shrinkage during fixation. Mar Ecol Prog Ser 110(2-3):293-299

Strom SL, Morello TA (1998) Comparative growth rates and yields of ciliates and heterotrophic dinoflagellates. J Plankton Res 20(3):571-584

Tada K, Pithakpol S, Yano R, Montani S (2000) Carbon and nitrogen content of Noctiluca scintillans in the Seto Inland Sea, Japan. J Plankton Res 22(6):1203-1211

Tang KW, Taal M (2005) Trophic modification of food quality by heterotrophic protists: species-specific effects on copepod egg production and egg hatching. J Exp Mar Biol Ecol 318(1):85-98

Throndsen J (1978) Preservation and storage. In: Sournia A (ed) Phytoplankton manual. UNESCO, Paris, pp 69-74

Tillmann U (2004) Interactions between planctonic microalgae and protozoan grazers. J Eukaryot Microbiol 51(2):156-168

Tomas CR (1996) Identifying marine diatoms and dinoflagellates. Acedemic Press Inc., San Diego, New York

Utermöhl H (1958) Zur Vervollkommnung der quantitativen Plankton-Methodik. Mitt Int Ver Theor Angew Limnol 9:1-38

Wiltshire KH, Dürselen CD (2004) Revision and quality analyses of the Helgoland Reede long-term phytoplankton data archive. Helgol Mar Res 58(4):252-268 
Wiltshire KH, Manly BFJ (2004) The warming trend at Helgoland Roads, North Sea: phytoplankton response. Helgol Mar Res 58:269-273

Wiltshire KH, Malzahn AM, Wirtz K, Greve W, Janisch S, Mangelsdorf P, Manly BFJ, Boersma M (2008) Resilience of North Sea phytoplankton spring bloom dynamics: an analysis of long-term data at Helgoland Roads. Limnol Oceanogr 53(4):1294-1302

Wirtz KW, Wiltshire K (2005) Long-term shifts in marine ecosystem functioning detected by inverse modeling of the Helgoland Roads time-series. J Mar Syst 56(3-4):262-282 